\title{
On modeling on a single-winding forced valve electromagnet in a circuit with a ballast resistor
}

\author{
Ye. I. Gracheva ${ }^{1}$, V. N. Petrov, ${ }^{2, *}, N$. V. Russova ${ }^{2}$, D. V. Samuilov ${ }^{2}$, G. P. Svintsov ${ }^{2}$, and I.V. Protosovitski ${ }^{3}$ \\ ${ }^{1}$ Kazan State Energy University, Kazan, Russia \\ ${ }^{2}$ Chivashsky State University named after I.N. Ulyanov, Cheboksary, Russia \\ ${ }^{3}$ Belarusian State Agrarian Technical University, Minsk, Belarus
}

\begin{abstract}
We have built a mathematical model of starting up a forced U-shaped valve electromagnet of static voltage taking into account dynamic parameters and characteristics. The offered model takes into account the influence of eddy currents in the closed circuits of the magnetic system. At the first stage we have developed a mathematical model that allows to determine optimum dimensions, to minimize the mass of active materials (the total mass of copper for windings and ferromagnetic steel), that are used in construction of the electromagnet. Then, based on a known method of integration of the dynamic equations we have obtained an actuation model of the valve magnet taking onto account equivalent resistances of cores of both circular and rectangular cross-sections. Modeling of dynamic parameters and characteristics was done taking into account secondary con-tours without increasing order of differential equations. We list calculated values of dynamic parameters and characteristics of the electromagnet in a circuit with a balance resistor.
\end{abstract}

\section{Introduction}

Modeling of dynamic parameters and characteristics is done [1] consequently in two stages. At the first stage, we determine geometric parameters and parameters of the magnetic system based on statistics while optimizing according to specific and generalized quality criteria [2]. At the second stage, we assess dynamic parameters and evaluate dynamic characteristics of the forced electric magnet. Further, we review a method for modeling dynamic parameters and characteristics of a singlewinding forced valve electromagnet in a circuit with a balance resistor.

\section{Methods}

Based on experimental data, B.V. Klimenko has earlier demonstrated [3] that during power supply of electromagnet winding with a secondary circuit they can be replaced with bifilar windings with full magnetic coupling.

Traction electromagnetic force, load characteristic, magnetization curve of the magnetic system of an electromagnet are described in $[4,5]$ in parametric form.

The assumption of the full magnetic coupling of the magnetizing winding and the secondary circuit allows us to record the total magnetomotive force:

$$
F=i \cdot N+i_{3} \cdot N_{3}
$$

Taking into account the assumption of the possibility of replacing the contours of eddy currents and other secondary contours with an equivalent short-circuited contour, and of a full magnetic coupling for the magnetizing winding and a secondary contour, the system of dynamic equations becomes

$$
\begin{gathered}
U=i \cdot R+\frac{d \psi}{d t} \\
0=i_{3} \cdot R_{3}+\frac{d \psi}{d t} \\
m_{\mathrm{red}} \frac{d V}{d t}=P_{\mathrm{em}}-P_{\mathrm{mec}} \\
\frac{d x}{d t}=V
\end{gathered}
$$

where $\mathrm{U}$ - voltage applied to the magnetizing winding; $\mathrm{R}$, i, N - resistance, current, number of turns of magnetizing winding; R3, i3, N3 - resistance, current, number of turns of the secondary circuit; $\Psi$ - instantaneous value of the winding flux linkage; mred - reduced mass of the moving electromagnet system; $\mathrm{V}$ - re-duced speed of the moving system; Pem - electromagnetic force; Pmec - mechanical force.

The drawing of the mechanical system with key dimensions can be seen in Figure 1.

\footnotetext{
* Corresponding author: pvn.chuvsu@yandex.ru
} 


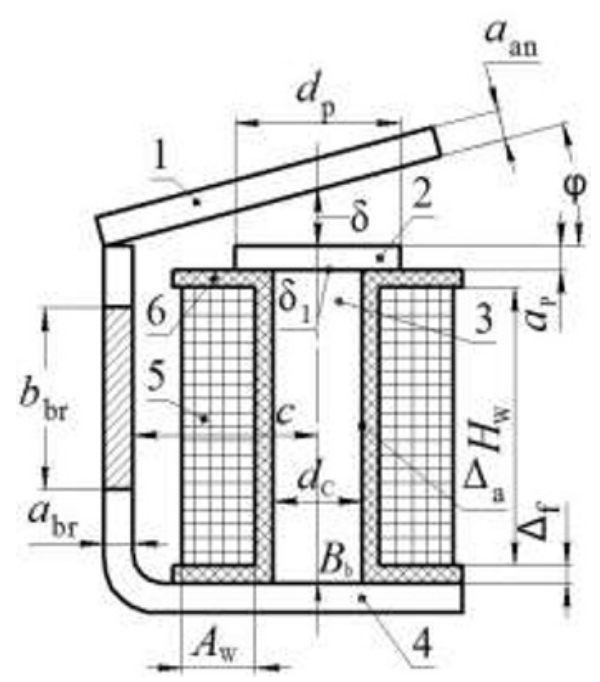

Fig. 1. The draft of a simplified magnetic scheme of the valve electromagnet.

The substitution scheme of the electromagnet's circuit in question can be seen on the Figure 2.

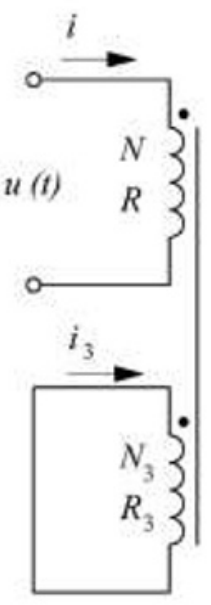

a

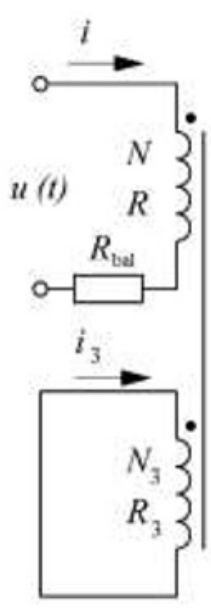

$\mathrm{b}$
Fig. 2. The substitution scheme of the electromagnet's circuit before (a) and after (b) the inclusion of a ballast resistor.

The scheme contains four secondary contours: a cylindrical core with resistance $R_{3.1}$, a round pole tip with resistance $R_{3.2}$, anchor with resistance $R_{3.3}$ and an Lshaped magnetic bracket with resistance $R_{3.4}$ (both the anchor and the bracket have rectangular cross-section). Then respective equivalent resistances of the circuits will equal [1]:

$$
\begin{aligned}
& R_{\mathrm{e} q 3.1}=\frac{8 \cdot \rho_{\mathrm{st.h}} \cdot \pi}{a_{\mathrm{p} . \mathrm{opt}}} ; R_{\mathrm{e} q 3.2}=\frac{8 \cdot \rho_{\mathrm{st.h}} \cdot \pi}{l_{\mathrm{c}}} \\
& R_{\text {eq } 3.3}=\frac{16 \cdot \rho_{\text {st.h }}}{l_{\text {an }}}\left(\frac{a_{\text {an.opt }}}{b_{\text {an.opt }}}+\frac{b_{\text {an.opt }}}{a_{\text {an.opt }}}\right) \\
& R_{e q 3.4}=\frac{16 \cdot \rho_{\mathrm{st} . \mathrm{h}}}{l_{\mathrm{br}}} \cdot\left(\frac{a_{\mathrm{br} . \mathrm{opt}}}{b_{\mathrm{br} . \mathrm{opt}}}+\frac{b_{\mathrm{br} . \mathrm{opt}}}{a_{\mathrm{br} . \mathrm{opt}}}\right)
\end{aligned}
$$

where $\quad \rho_{\text {st.h }}=\rho_{\text {st. } 0} \cdot\left(1+\alpha_{\text {st }} \cdot 0,8 \cdot \Theta_{V}\right)$ is electrical resistivity of steel when the electromagnet is in a heated state; $a_{\text {p.opt }}$ is length of an average magnetic circuit at the pole tip; $l_{\mathrm{c}}=H_{\mathrm{w} . \mathrm{opt}}+2 \cdot \Delta_{\mathrm{f} . \mathrm{opt}}$ is length of an average magnetic circuit at the core; $l_{\mathrm{an}}=c_{\mathrm{opt}}+a_{\mathrm{br} . \mathrm{opt}}$ is length of an average force line at the anchor; $l_{\text {br }}=c_{\text {opt }}+a_{\text {br.opt }}+0,5 d_{\text {c.o.oo }}+a_{\text {p.opt }}+H_{\text {w.opt }}+2 \cdot \Delta_{\text {f.opt }}$ is length of an average force line at the L-shaped magnetic bracket.

The expressions listed can be replaced with one equivalent single-circuit resistance

$$
R_{\mathrm{eq} 3}=\left(\sum_{i=1}^{n} \frac{N_{i}^{2}}{R_{\mathrm{eq} 3 i}}\right)^{-1}
$$

where $N_{i}, R_{\text {eq } 3 i}$ is number of loops and resistance of the $i$-th short-circuited contour; $n$ is number of contours.

Then, after substituting, the expression (6) will be:

$$
R_{\text {eq } 3}=\left(\frac{a_{\text {p.opt }}+l_{\mathrm{c}}}{8 \cdot \rho_{\text {st.h }} \cdot \pi}+\frac{l_{\text {an }}+l_{\text {br }}}{16 \cdot \rho_{\text {st.h }} \cdot\left(a_{\text {br.opt }} / b_{\text {br.opt }}+b_{\text {br.opt }} / a_{\text {br.opt }}\right)}\right)^{-1}
$$

The equations (2) and (3) allow to obtain the connection between the currents of the magnetizing winding and in the secondary circuit

$$
U=i \cdot R-\frac{N}{N_{3}} \cdot i_{3} \cdot R_{\mathrm{eq} 3}
$$

Solving together the equations (1) and (8) we can establish the connection between the currents in the circuits and magnetomotive force

$$
\begin{gathered}
i=\frac{U+R_{\mathrm{eq} 3} \cdot F / N}{R+R_{\mathrm{eq} 3}{ }^{\prime}} \\
i_{3}=\frac{F-i / N}{N_{3}}
\end{gathered}
$$

where $R_{\mathrm{E} 3}{ }^{\prime}=R_{\mathrm{E} 3} \cdot N^{2} / N_{3}{ }^{2}$ is equivalent reduced resistance of the secondary single-loop circuit.

Then, it is enough to integrate one of the equations of the system of the first or second contour, for example, the expression (2).

Substituting (9) into (2), the system of the dynamic differential equations can be written as follows:

$$
\begin{gathered}
\frac{d \psi}{d t}=\frac{R_{\mathrm{eq} 3}{ }^{\prime}}{R+R_{\mathrm{eq} 3}{ }^{\prime}}(U-R \cdot F / N) \\
m_{\mathrm{red}} \frac{d V}{d t}=P_{\mathrm{em}}-P_{\mathrm{mec}}
\end{gathered}
$$




$$
\frac{d x}{d t}=V
$$

Neglecting residual induction (for mild steel grade 10895), the initial value of flux linkage is taken to be zero, i.e. $\Psi(0)=0$.

The starting speed of the anchor and its travel distance equal zero $(V(0)=0 ; x(0)=0)$.

The first initial condition determines the starting value of the magnetomotive force of the windings $(F=0)$. Thus, we can observe rapid changes in currents in the magnetizing windings and in the short-circuited contour at $\mathrm{t}=0$

$$
i(0)=\frac{U}{R+R_{\mathrm{eq} 3}} ; i_{3}(0)=\frac{i(0) \cdot N}{N_{3}} .
$$

During the startup time, the anchor of the electromagnet is static (its speed and travel distance equal zero). After it is actuated (the anchor moves to the final position), the anchor speed also equals zero, and the distance travelled is a difference between the starting and end positions of the working air gap. In order to ensure these conditions, we are introducing the $\xi$ coefficient. The coefficient equals one if the anchor is moving and zero when it is static.

Finally, the system of differential equations can be written in the form convenient for solving using Euler's method [6]:

$$
\begin{aligned}
& \Psi_{k+1}=\Psi_{k}+\left(U-i_{k} \cdot R\right) \cdot \Delta t \\
& V_{k+1}=\xi \cdot\left(V_{k}+\frac{P_{\mathrm{em}_{k}}-P_{\operatorname{mec}_{k}}}{m_{\mathrm{red}}} \cdot \Delta t\right) \\
& x_{k+1}=x_{k}+\xi \cdot V_{k} \cdot \Delta t
\end{aligned}
$$

where $\Psi \mathrm{k}+1, \mathrm{Vk}+1, \mathrm{xk}+1$ - accordingly, flux linking, speed and displacement at the beginning of the $(\mathrm{k}+1)$-th segment;

$\Psi \mathrm{k}, \mathrm{Vk}, \mathrm{xk}$ - accordingly, flux linking, speed and displacement at the beginning of the k-th segment;

$\mathrm{ik}$, Pem k, Pmec k - respectively, the current in the magnetizing winding, electromagnetic and opposing forces at the beginning of the k-th section;

$\Delta \mathrm{t}$ - integration step.

In order to calculate according to the expressions (14-16) we need to obtain the following values for every section:

1) averaged value of magnetic induction in the crosssection of the core base $B_{\mathrm{b} k}$ :

$$
B_{\mathrm{b} k}=\frac{4}{N \cdot \pi \cdot d_{\mathrm{c} . \mathrm{opt}}^{2}} \cdot \frac{\Psi_{k}}{\Psi_{*_{k}}}
$$

2) magnetomotive force of the winding $F_{k}$ : $F_{k}=\left\{\begin{array}{l}F_{*}\left(B_{\mathrm{b} . \text { boundary }}\right) \cdot F_{\mathrm{bas}}\left(B_{\mathrm{o} k}\right) \text { при } B_{\mathrm{b} k} \leq B_{\mathrm{b} . \text { boundary }} \\ F_{*}\left(B_{\mathrm{b} k}\right) \cdot F_{\mathrm{bas}}\left(B_{\mathrm{o} k}\right) \text { при } B_{\mathrm{b} k}>B_{\mathrm{b} . \text { boundary }}\end{array}\right.$
3) electromagnetic moment $M_{\mathrm{em} k}$ and force $P_{\mathrm{em} k}$ :

$$
\begin{gathered}
M_{\mathrm{em} k}=\left\{\begin{array}{l}
M_{*}\left(B_{\mathrm{b} . \mathrm{boundary}}\right) \cdot M_{\mathrm{bas}}\left(B_{\mathrm{b} k}\right) \text { при } B_{\mathrm{b} k} \leq B_{\mathrm{b} . b o u n d a r y} ; \\
M_{*}\left(B_{\mathrm{b} k}\right) \cdot M_{\mathrm{bas}}\left(B_{\mathrm{b} k}\right) \text { при } B_{\mathrm{b} k}>B_{\mathrm{b} . \text { boundary }} ;
\end{array}\right. \\
P_{\mathrm{e} m k}=\frac{M_{\mathrm{em} k}}{d_{\mathrm{c} . \mathrm{opt}} \cdot\left(c_{\mathrm{opt} *}+a_{\mathrm{br} . \mathrm{opt} *}\right)}
\end{gathered}
$$

The expressions (18) and (19) are obtained on the basis of experimental research of load testing of valve electromagnets $[5,7]$.

The currents in the winding and in the secondary contour are calculated according to expressions (9) and (10), counteracting force $P_{\mathrm{em}} k-$ using a known dependency.

The reduced mass of the anchor equals to

$$
m_{\text {an.red }}=\frac{J}{\left(d_{\text {c.opt }} \cdot\left(c_{\mathrm{opt} *}+a_{\mathrm{br} . \mathrm{opt} *}\right)\right)^{2}}
$$

where $J$ is the moment of inertia of the moving system relative to the rotation axis of the electromagnet's anchor:

$$
\begin{gathered}
J=m_{\mathrm{an}} \cdot \frac{l_{\mathrm{an}}{ }^{2}}{3} \\
l_{\mathrm{an}}=c_{\mathrm{opt}}+a_{\mathrm{br} . \mathrm{opt}}+0,5 d_{\mathrm{c} . \mathrm{opt}}+A_{\mathrm{w} . \mathrm{opt}}+2 \cdot \Delta_{\mathrm{f} . \mathrm{opt}}
\end{gathered}
$$

The reduced mass of the moving system depends on the kinetic system of the contactor and on the mass of intermediate links and is assumed to be on average 1,4 times higher than the reduced mass of the anchor:

$$
m_{\text {red }}=1,4 \cdot m_{\text {an.red }}
$$

During the opening of the forcing contact, the add-on resistance of the ballast resistor is introduced into the electromagnet power circuit and the resistance of the primary circuit can be written in the form of (23):

$$
R=\left\{\begin{array}{l}
R_{\mathrm{h}} \text { при } x_{k} \leq x_{\text {rel }} ; \\
R_{\mathrm{h}}+R_{\mathrm{bal}} \text { при } x_{k}>x_{\mathrm{rel}},
\end{array}\right.
$$

where $x_{\text {rel }}$ is a coordinate of opening of the forcing contact (see Figure 3).

The electromagnetic time constant $T$ of the rise of the magnetomotive force can be determined using the expression [3]:

$$
T=N^{2} \cdot \Lambda_{\mathrm{i}} \cdot \frac{R+R_{\mathrm{e} q 3}{ }^{\prime}}{R \cdot R_{\mathrm{e} q 3}{ }^{\prime}}
$$

where $\Lambda_{\mathrm{i}}$ is reduced conductivity of the magnetic system with starting gap of $\delta_{\mathrm{i}}\left(\delta=\delta_{\mathrm{i}}\right)$. 


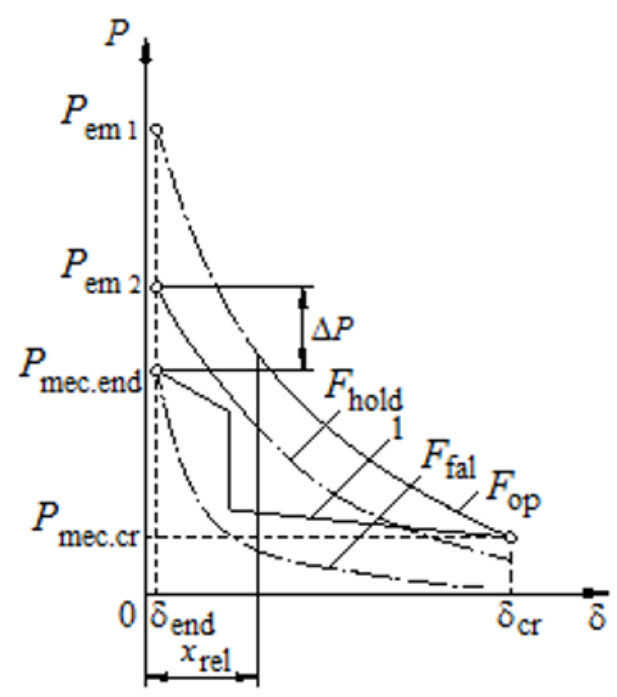

Fig. 3. Mechanical (1) and traction characteristics electromagnet.

Other methods exist as well. For instance, methods of modern solutions of modeling equations and dynamic processes. However, those are very time-consuming $[8$, 9].

Dynamic characteristics can be calculated after determining the size of the magnetic system of the electromagnet during its design and optimization. During that time, source data are: material of the magnet line (steel of the 10895 grade), parameters of the mechanical characteristic (critical value of the mechanical characteristic, the relation between the final value of the mechanical force to the critical value $P_{\text {mec.end*A), }}$ ) temperature of the environment $T_{0}$ and permissible value of heating temperature $\Theta_{\text {per, }}$, the multiplicity of the maximum voltage in fractions of the operating voltage, the filling factor of the winding window; and for calculating the dynamic parameters along with the above initial data we require: a family of load characteristics and magnetization curves.

\section{Results}

In order to illustrate the results of the modeling according to the method described above we list static and dynamic parameters of the electromagnet in the tables $1-4$ for the following source data: $\Theta$ per $=155{ }^{\circ} \mathrm{C}$, Tam $=70{ }^{\circ} \mathrm{C}$, Pmec.cr $=10 \mathrm{H}$, Pmec.end $=4 \cdot$ Pmec. $. \mathrm{cr}=40$ $\mathrm{H}, \delta \mathrm{i}=16 \mathrm{~mm}(\varphi \approx 12$ degrees $)$.

In the tables 2-4 we assume the following denominations: core diameter, winding height, winding thickness, distance from the core axis to the magnetic bracket, flat tip diameter, bracket thickness, anchor thickness, bracket width, anchor width, flat tip thickness, coil frame thickness, air gap width, magnetomotive force of the active winding, magnetomotive force of the holding winding, magnetomotive force of the falling winding, activation power, holding power of the winding in hold mode, power of the winding and ballast resistor in hold mode , electromagnetic force upon actuation, electromagnetic force on hold, mass of active materials, induction at the base of the core upon actuation, induction at the base of the core when the armature enters the final position, induction at the base of the core on hold induction at the base of the core in hold mode, induction when the anchor falls off at the base of the core, induction when the anchor falls off in the working gap, pulling time, boost time, main contact closure time, response time, armature speed when triggered, transient duration, electromagnetic time constant, overvoltage on the winding at the moment of switching on the ballast resistor, nominal voltage on the winding, diameter of the copper wire of the winding, fill factor of the copper wire, number of turns in the wires, the resistance of the winding in the heated state, the resistance of the ballast resistor.

\section{Discussions}

Thus, the method listed above allows us to calculate the dynamic parameters of the electromagnet in the control

Table 1. Geometric dimensions of the electromagnet.

\begin{tabular}{|c|c|c|c|c|c|c|c|c|c|}
\hline $\begin{array}{c}d_{\mathrm{c}}, \\
\mathrm{mm}\end{array}$ & $\begin{array}{c}H_{\mathrm{w}}, \\
\mathrm{mm}\end{array}$ & $\begin{array}{c}A_{\mathrm{w}}, \\
\mathrm{mm}\end{array}$ & $\begin{array}{c}c, \\
\mathrm{~mm}\end{array}$ & $\begin{array}{c}d_{\mathrm{p}}, \\
\mathrm{mm}\end{array}$ & $\begin{array}{c}a_{\mathrm{br}}=a_{\mathrm{an}}, \\
\mathrm{mm}\end{array}$ & $\begin{array}{c}b_{\mathrm{br}}=b_{\mathrm{an}}, \\
\mathrm{mm}\end{array}$ & $\begin{array}{c}a_{\mathrm{p}}, \\
\mathrm{mm}\end{array}$ & $\begin{array}{c}\Delta_{\mathrm{f}}, \\
\mathrm{mm}\end{array}$ & $\begin{array}{c}\Delta_{\mathrm{a}}, \\
\mathrm{mm}\end{array}$ \\
\hline 33,1 & 38,7 & 15,7 & 67,1 & 55 & 8,3 & 104 & 13,2 & 3,3 & 0,83 \\
\hline
\end{tabular}

Table 2. Static parameters of the electromagnet.

\begin{tabular}{|c|c|c|c|c|c|c|c|c|c|c|c|c|c|}
\hline $\begin{array}{c}F_{\text {op }}, \\
\text { A }\end{array}$ & $\begin{array}{c}F_{\text {hold, }} \\
\mathrm{A}\end{array}$ & $\begin{array}{c}F_{\text {fal }}, \\
\mathrm{A}\end{array}$ & $\begin{array}{c}P_{\text {op }}, \\
\mathrm{W}\end{array}$ & $\begin{array}{c}P_{\text {hold(wind.) }}, \\
\mathrm{W}\end{array}$ & $\begin{array}{c}P_{\text {hold(wind. }+R \text { bal) }}, \\
\mathrm{W}\end{array}$ & $\begin{array}{c}P_{\text {em.op }}, \\
\mathrm{N}\end{array}$ & $\begin{array}{c}P_{\text {em.hold, }}, \\
\mathrm{N}\end{array}$ & $\begin{array}{c}M_{\text {a }} \\
\mathrm{kg}\end{array}$ & $\begin{array}{c}B \text { b.op, } \\
\mathrm{T}\end{array}$ & $\begin{array}{c}B \text { b.op.end, } \\
\mathrm{T}\end{array}$ & $\begin{array}{c}B \text { b.hold, } \\
\mathrm{T}\end{array}$ & $\begin{array}{c}B \text { b.fal2, } \\
\mathrm{T}\end{array}$ & $\begin{array}{c}B \text { b.fall, } \\
\mathrm{T}\end{array}$ \\
\hline 1857 & 856 & 90 & 131 & 27,9 & 60,5 & 122,6 & 74,2 & 2,674 & 1,132 & 1,65 & 1,204 & 0,342 & 0,206 \\
\hline
\end{tabular}

Table 3. Dynamic parameters of the electromagnet.

\begin{tabular}{|c|c|c|c|c|c|c|c|}
\hline $\begin{array}{c}t_{\text {start}}, \\
\mathrm{ms}\end{array}$ & $\begin{array}{c}t_{\text {rel }}, \\
\mathrm{ms}\end{array}$ & $\begin{array}{c}t_{\mathrm{m} . \mathrm{c},}, \\
\mathrm{ms}\end{array}$ & $\begin{array}{c}t_{\mathrm{op}}, \\
\mathrm{ms}\end{array}$ & $v_{\mathrm{op}}, \mathrm{m} / \mathrm{s}$ & $t_{\mathrm{tp}}, \mathrm{ms}$ & $\tau, \mathrm{ms}$ & $\begin{array}{c}d U, \\
\mathrm{~V}\end{array}$ \\
\hline 76,8 & 148,6 & 151,3 & 157,7 & 0,84 & 306,5 & 127 & 142 \\
\hline
\end{tabular}

Table 4. Parameters of the winding and ballast resistor.

\begin{tabular}{|c|c|c|c|c|c|}
\hline$U_{\text {rat }}, \mathrm{V}$ & $d_{\text {cop }}, \mathrm{mm}$ & $K_{\text {cop }}$ & $N$ & $R_{\mathrm{h},}, \Omega$ & $R_{\text {bal }, ~} \Omega$ \\
\hline 220 & 0,28 & 0,572 & 5630 & 422 & 494 \\
\hline
\end{tabular}


circuit with a ballast resistor, which is has found broad use due to the simplicity of implementation. The main aspects of the method can be used to develop a design method for the electromagnet with pulse-width modulated winding voltage.

\section{Conclusions}

1. If it is necessary to increase the speed of the electromagnet, it can be achieved by increasing the starting power in the operation mode. To ensure the heating of the winding is not higher than the permissible temperature in the holding mode (with a constant cooling area), we recommend to change the power dissipated by the ballast resistor.

2 . The proposed calculation procedure makes it possible to take into account the influence of the supply voltage values on the dynamic parameters of the electromagnet by varying the resistance of the ballast resistor while keeping the winding resistance constant.

3 . We present the results of calculating the geometric dimensions and parameters of an electromagnet minimized by the mass of active materials under static conditions. The basic dynamic parameters are calculated, as well as the parameters of the winding and ballast.

\section{References}

[1] B.V. Klimenko, Forced electromagnetic systems. Moscow, Energoatomizdat Publ.,160 (1989)

[2] V.N. Petrov, N.V. Russova, D.V. Samuilov, G.P. Svintsov, Results of designing a forced DC solenoid electromagnet in a circuit with a ballast resistor. Bulletin of the Chuvash University, 1, pp. 91-101 (2019).

[3] B.V. Klimenko, Integration of the equations of electromagnet dynamics in the presence of secondary circuits. Electricity, 11, pp. 52-55 (1984).

[4] Yu.M. Zaitsev, V.N. Petrov, N.V. Russova, G.P. Svintsov, Method of synthesis of a forced DC valve magnet electromagnet in a circuit with a ballast resistor. Bulletin of the Chuvash University, 1, pp. 103-112 (2017).

[5] V.K. Kadykov, N.V. Russova, G.P. Svintsov, A.V. Sizov, Obobshchennye eksperimental'nye zavisimosti potokoraspredeleniya, potokoscepleniya i magnitodvizhushchej sily v klapannyh elektromagnitnyh sistemah postoyannogo toka s kruglymi polyusnymi nakonechnikami. Elektrotekhnika, 4, pp. 41-47 (2007).

[6] V.P. D'yakonov, Handbook of algorithms and programs in the BASIC language for personal computers. Moscow, Nauka Publ.,240 (1987)

[7] V.K. Kadykov, To the synthesis of optimal drive electromagnets contactors for low-voltage complete devices, Electrical Engineering 2010: collection of reports of the VII Symposium, Moscow region, May 27 29, 2003. Moscow, 2, pp. 237-240 (2003).

[8] A.G. Nikitenko, V.G. Shcherbakov, B.N. Lobov, L.S. Lobanova. Mathematical modeling and design automation traction electrical apparatuses. Moscow, Vysshaya shkola Publ., 537, (1996).
[9] B.N. Lobov, To the calculation of the characteristics of electromagnetic drives of electrical apparatus. Izv. Vyssh. Uchebn. Zaved., Elektromekh., 60(1), pp. 35-40 (2017). 